\title{
Generalized Spring Tensor Algorithms: with Workflow Scheduling Applications in Cloud Computing
}

\author{
Shahrzad Aslanzadeh \\ University of Technology Sydney \\ Center of Real-time Information Networks
}

\author{
Zenon Chaczko \\ University of Technology Sydney \\ Center of Real-time Information Networks
}

\begin{abstract}
In Cloud Computing, designing an efficient workflow scheduling algorithm is considered as a main goal. Load balancing is one of the most sophisticated methodologies, which can optimize workflow scheduling by distributing the load evenly among available resources. A well-designed load balancing algorithm has significant impact on performance and output in Cloud Computing. Therefore, designing robust load balancing techniques to manage the networks' load has always been a priority. Researchers have proposed and examined different load balancing methods; there is, however, a large knowledge gap in adopting an efficient load balancing algorithm in the Cloud system.
\end{abstract}

This paper describes how a generalized spring tensor, an evolutionary algorithm with mathematical apparatus, can be utilized for a more efficient and effective load management in Cloud Computing. Considering the fluctuation and magnitude of the load, a novel application of workflow scheduling is investigated in the context of various mathematical patterns. The preliminary results of the research show that defining the dependency ratio between workflow tasks in Cloud Computing, results in better resource management, maximized performance and minimized response time while dealing with customer's requests.

\section{General Terms}

Cloud Computing; Load balancing

\section{Keywords}

Cloud Computing; Load balancing; Evolutionary algorithm; Workflow scheduling

\section{INTRODUCTION}

Cloud Computing is composed of several different resources, interconnected to each other to form a network or a grid. These resources should be flexible and dynamic in terms of usage and allocations.

The term "availability", was always a main concern in CloudComputing. Fundamentally, availability explains the ubiquitousness of the network information in case of resource scaling $[1,2]$. In the Cloud system, load balancing is one of the major techniques that has a dramatic impact on resource availability. Today, most of the Cloud vendors are trying to use an automated load balancer to scale the numbers of the resources automatically when providing the users with access to computation services. Promoting the availability and performance of the Cloud system highlights the main goal of the automated load balancers.

Various types of load balancing techniques offered to manage the load changes in the Cloud system. Workflow scheduling, however, is attracting more attention as there is a gap in resource availability and reliability verifications. [3][4]. Workflow scheduling is suitable for those types of tasks with workflow structured patterns. In these models, each job is composed of several dependent tasks. Therefore execution of one task is dependent on another [5]. Failures in tasks' execution, affect the performance of the whole system. Currently there is a lack of effective algorithms which deal with load balancing on workflow structured jobs [6].

This paper describes, how an evolutionary algorithm, specifically generalized spring tensor model (STeM), can be adopted to schedule the workflow structured jobs on available resources. Possible ways to improve the performance of the Cloud system, by applying the mathematical patterns of the STeM algorithm will be discussed.

\section{WORKFLOWSCHEDULING}

Reviewing the literature, various load balancing algorithms are proposed. Tao[7] and Suraj [8] designed a workflow load balancing algorithm based on particle swarm optimization. Considering the robustness characteristic, the algorithm mainly focuses on costs minimizations. Timur [9] optimized the Ant Colony algorithm to minimize the average relative percentage of imbalance in workflow scheduling. Jindadun [10] applied a hybrid technique in a private Cloud to minimize the response time in resource scheduling.

Genetic Algorithms (GAs) also have been considered as a workflow load balancing technique which allows to reduce the number of the virtual machines [11].

The SHEFT workflow algorithm is well known for workflow load balancing scheduling, as it is optimizing the execution time, while ensuring elasticity in the Cloud network [12].

Beside the benefits of the above mentioned algorithms, there are still challenges regarding the availability and reliability of the Cloud [3, 13]. As load changes dynamically, it is difficult to manage workflow scheduling and optimization simultaneously[14].

\section{PROPOSED MODEL}

Different computational algorithms have been suggested to analyze the dynamics and complexity of the Cloud. The Coarse-gained algorithm is one of the most prominent methods, developed to study the complication of the elastic networks [15]. A Generalized Spring Tensor algorithm categorized as a coarse-gained technique is able to manage the network complexity by analyzing the magnitude and the direction of the load.

Fundamentally, the STeM algorithm is inspired by the Anisotropic Network Model (ENM) and Gaussian Network Model (GNM). The ANM is suited to predict the direction of the motions and it is fundamentally based on the Hookean law. Hooke's law is a principle in physics, which is elaborating the relation between force and movement of the elastic bodies. Force $\mathrm{P}$ moves the elastic body from its relaxed state $\left(d_{1}\right)$ to a new position $\left(d_{2}\right)$ by $\Delta$ d. Stiffness of the elastic body changes the maximum movement, resulted from force $P$. The ANM, however; is not accurate for predicting the 
magnitude of the force between two nodes. The GNM, conversely, was introduced to study the fluctuation of the magnitude. However, as it is only applicable for isotropic

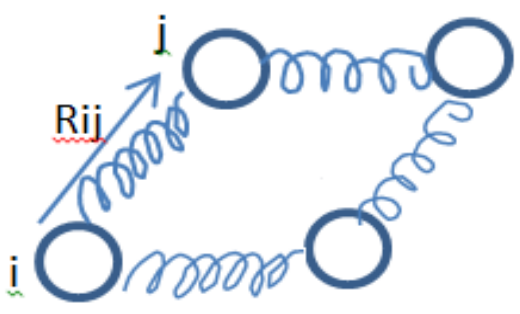

Figure 1-Representation of dependent tasks in Elastic mass spring model

models, it's not useful for predicting the direction of the magnitude between nodes. Bahar et al [15] in their studies, combined two models of GNM and ANM, and named it as STeM. Using the Go-like algorithm as the potential model, their proposed algorithm highlights the direction and fluctuation of the magnitude between different nodes in network [16].

In order to manage the load efficiently, while decreasing the failure impact on the system, in this paper, we are proposing a new application, which finds the magnitude and direction of the Cloud load. Using the STeM algorithm, this application will help the system architects to analyze the direction and magnitude of the load, between dependent tasks. "Fig. 1 " is modelling two dependent tasks $\mathrm{i}$ and $\mathrm{j}$ in Cloud environment, where $S_{i}=\left(x_{i}, y_{i}, z_{i}\right)$ and $S_{j}=\left(x_{j}, y_{j}, z_{j}\right)$.

Adapting Hookean law principles, in this research, we are assuming that a "job" is a collection of multiple dependent tasks that are connected to each other by characteristics such as memory usage, start and finish time [17]. By applying the STeM algorithm, the correlation between the tasks $i$ and $j$ will be calculated. STeM algorithm uses the $3 \mathrm{~N} \times 3 \mathrm{~N}$ Hessian matrix, which is then composed of four $3 \times 3$ matrices. These matrices determine the bond bending, angles, torsion forces and the non-local interaction between tasks $\mathrm{i}$ and $\mathrm{j}$. Therefore, each Hessian matrix is defined as follows:

$$
\begin{aligned}
& \frac{\partial^{2} \mathrm{~V}_{1\left(\mathrm{r}, \mathrm{r}_{0}\right)}}{\partial \mathrm{X}_{\mathrm{i}} \mathrm{X}_{\mathrm{j}}} \quad \frac{\partial^{2} \mathrm{~V}_{1\left(\mathrm{r}, \mathrm{r}_{0}\right)}}{\partial \mathrm{X}_{\mathrm{i}} \mathrm{X}_{\mathrm{j}}} \quad \frac{\partial^{2} \mathrm{~V}_{1\left(\mathrm{r}, \mathrm{r}_{0}\right)}}{\partial \mathrm{X}_{\mathrm{i}} \mathrm{Z}_{\mathrm{j}}} \\
& \mathrm{H}_{\mathrm{ij}}=\left[\begin{array}{lll}
\frac{\partial^{2} \mathrm{~V}_{1\left(\mathrm{r}, \mathrm{r}_{0}\right)}}{\partial \mathrm{XY}_{\mathrm{i}} \mathrm{X}_{\mathrm{j}}} & \left.\frac{\partial^{2} \mathrm{~V}_{1\left(\mathrm{r}, \mathrm{r}_{0}\right)}}{\partial \mathrm{Y}_{\mathrm{i}} \mathrm{Y}_{\mathrm{j}}} \quad \frac{\partial^{2} \mathrm{~V}_{1\left(\mathrm{r}, \mathrm{r}_{0}\right)}}{\partial \mathrm{Y}_{\mathrm{i}} \mathrm{Z}_{\mathrm{j}}}\right]
\end{array}\right] \\
& \frac{\partial^{2} \mathrm{~V}_{1\left(\mathrm{r}, \mathrm{r}_{0}\right)}}{\partial \mathrm{Z}_{\mathrm{i}} \mathrm{X}_{\mathrm{j}}} \frac{\partial^{2} \mathrm{~V}_{1\left(\mathrm{r}, \mathrm{r}_{0}\right)}}{\partial \mathrm{Z}_{\mathrm{i}} \mathrm{Y}_{\mathrm{j}}} \quad \frac{\partial^{2} \mathrm{~V}_{1\left(\mathrm{r}, \mathrm{r}_{0}\right)}}{\partial \mathrm{Z}_{\mathrm{i}} \mathrm{Z}_{\mathrm{j}}}
\end{aligned}
$$

The value of the four above mentioned matrices will be calculated and based the stiffness of the springs, the correlation between $\mathrm{i}$ and $\mathrm{j}$ explains:

$<\Delta \mathrm{r}_{\mathrm{i}} \cdot \Delta \mathrm{r}_{\mathrm{j}}>=\frac{3 \mathrm{k}_{\beta} \mathrm{T}}{\mu}\left(\mathrm{H}_{3 \mathrm{i}-2,3 \mathrm{j}-2}^{+}+\mathrm{H}_{3 \mathrm{i}-1,3 \mathrm{j}-1}^{+}+\mathrm{H}_{3 \mathrm{i}, 3 \mathrm{j}}^{+}\right)$

$\mathrm{k}_{\beta} \mathrm{T}$ is the force $\mathrm{P}$ that should be applied on $\mathrm{i}$ and $\mathrm{j}$. The expected results highlight the task dependencies ratio in the Cloud network, which help in better resource management planning and recovery administration in case of any failure.

\subsection{Simulation and results}

The application of a generalized spring tensor is developed in Matlab. As a case study, a simulation of the STeM algorithm has been setup and evaluated on a cluster of dependent tasks. For each task resource characteristics such as memory usage, CPU usage, start and finishing times were investigated.

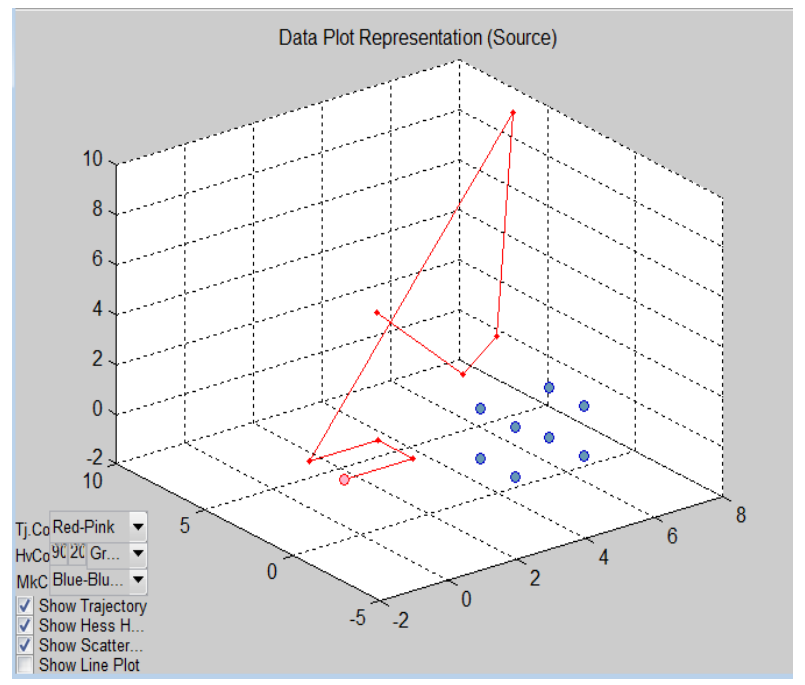

Figure 2- Data source plot representation

The values of these characteristics are used as a input data to model the behaviour of the imposed load on the Cloud network. Simulation tests indicate that in the Elastic Model of the Cloud Computing, tensor geometry identifies the fluctuation of the load on bounded mass spring model of the dependent tasks. "Fig.2" is showing the data source plotting, explaining the correlation of the tasks in 3D space. Simulation results with visual patterns make the load management easier, as the fluctuation and magnitude of the load can be observed in the whole network.

\section{CONCLUSION}

This paper presents an application of a Generalized Spring Tensor algorithm for managing workflow scheduling in the Cloud system. The approach allows the assessment of the direction and magnitude of the fluctuations in the network of mass springs (Cloud Computing resources). In order to examine the behaviour of the Cloud network, a large number of nodes are selected with the mass-spring tensor relations. The approach allows identifying and analyzing different mathematical patterns of various dependent tasks. The advantages of the method highlight the dependency ratio between workflow tasks, which result in better resource utilization and management, optimized performance and minimized response times. As a future work, different sets of task characteristics will be selected to analyze their correlation and impact on the Cloud network.

\section{REFERENCES}

[1] A. Gupta, O. Sarood, L. V Kale, and D. Milojicic, "Improving HPC Application Performance in the Cloud through Dynamic Load Balancing," in Cluster, the Cloud and Grid Computing (CCGrid), 2013 13th IEEE/ACM International Symposium on, 2013, pp. 402-409.

[2] P. Mathur and N. Nishchal, "Cloud computing: New challenge to the entire computer industry," in Parallel 
Distributed and Grid Computing (PDGC), 2010 1st International Conference on, 2010, pp. 223-228.

[3] L. Singh, "A Survey of Workflow Scheduling Algorithms and Research Issues," vol. 74, no. 15, pp. 2128, 2013.

[4] C. Zhang, H. De Sterck, M. Jaatun, G. Zhao, and C. Rong, "CloudWF: A Computational Workflow System for Clouds Based on Hadoop," in Cloud Computing, vol. 5931, 2009, pp. 393-404.

[5] S. Tilak and P. D. Patil, "A Survey of Various Scheduling Algorithms in Cloud Environment," vol. 1, no. 2, pp. 36-39, 2012.

[6] S. Shenai and Vijindra, "Survey on Scheduling Issues in Cloud Computing," Procedia Engineering, vol. 38. pp. 2881-2888, 2012.

[7] Q. Tao, H. Chang, Y. Yi, C. Gu, and Y. Yu, "QoS Constrained Grid Workflow Scheduling Optimization Based on a Novel PSO Algorithm," 2009 Eighth Int. Conf. Grid Coop. Comput., no. 60873162, pp. 153-159, Aug. 2009.

[8] S. Pandey, L. Wu, S. M. Guru, and R. Buyya, "A Particle Swarm Optimization-Based Heuristic for Scheduling Workflow Applications in Cloud Computing Environments," 2010 24th IEEE Int. Conf. Adv. Inf. Netw. Appl, pp. 400-407, 2010.

[9] T. Keskinturk, M. B. Yildirim, and M. Barut, "An ant colony optimization algorithm for load balancing in parallel machines with sequence-dependent setup times," Comput. Oper. Res., vol. 39, no. 6, pp. 1225-1235, Jun. 2012.
[10] J. Li and J. Peng, "An Energy-efficient Scheduling Approach Based on Private Clouds « VM Workflow Scheduling in Private Clouds," vol. 4, no. 10, pp. 716724, 2011.

[11] S. Sawant, "A Genetic Algorithm Scheduling Approach for Virtual Machine Resources in a Cloud Computing Environment," 2011.

[12] C. Lin and S. Lu, "Scheduling Scientific Workflows Elastically for Cloud Computing," 2011 IEEE 4th Int. Conf. Cloud Comput., pp. 746-747, 2011.

[13] Z. Chaczko and S. Aslanzadeh, "C2EN: Anisotropic Model of Cloud Computing," in Systems Engineering (ICSEng), 2011 21st International Conference on, 2011, pp. $467-473$.

[14] L. Wei, L. Shanping, and W. Xingen, "Load balance optimization with replication degree customization," in Cloud Computing and Intelligence Systems (CCIS), 2011 IEEE International Conference on, 2011, pp. 170-174.

[15] I. Bahar and A. J. Rader, "Coarse-grained normal mode analysis in structural biology," Curr. Opin. Struct. Biol., vol. 15 , no. 5, pp. 586-592, 2005.

[16] L. Tu-Liang and S. Guang, "Generalized spring tensor models for protein fluctuation dynamics and conformation changes," in Bioinformatics and Biomedicine Workshop, 2009. BIBMW 2009. IEEE International Conference on, 2009, pp. 136-143.

[17] Hooke's Law, Simple Harmonic Oscillator. MIT Course 8.01: Classical Mechanics, Lecture 10. Cambridge, MA USA: MIT OCW, 1999. 